\title{
Studies on structural, optical and electrical properties of electron beam evaporated $\mathrm{Cu}_{2} \mathrm{SnSe}_{3}$ thin films
}

\author{
P. Prathiba Jeya Helan ${ }^{1}$, K. Mohanraj ${ }^{1 *}$, G. SivakumaR $^{2}$ \\ ${ }^{1}$ Department of Physics, Manonmaniam Sundaranar University, Tirunelveli, Tamil Nadu, India \\ ${ }^{2}$ CISL, Department of Physics, Annamalai University, Annamalai Nagar, Tamil Nadu, India
}

\begin{abstract}
The present work describes the deposition of semiconducting $\mathrm{Cu}_{2} \mathrm{SnSe}_{3}$ thin films by electron beam evaporation method. The structure of the deposited films was characterized by XRD and Raman analysis. X-ray diffraction study revealed that the $\mathrm{Cu}_{2} \mathrm{SnSe}_{3}$ thin films had a cubic sphalerite-like structure with crystallite size of $12 \mathrm{~nm}$. Raman spectrum of the thin films confirmed the phase purity. FESEM analysis showed a continuous film with polydispersed grains of a diameter less than $1 \mu \mathrm{m}$ and the elemental composition was confirmed by EDS spectrum. The UV-Vis spectrum revealed that the sample had high absorption in the visible region and the band gap was found to be $1.15 \mathrm{eV}$. The I-V graph exhibited the electrical resistivity and conductivity of the film as $2.13 \Omega \cdot \mathrm{cm}$ and $0.468 \mathrm{~S} / \mathrm{cm}$, respectively. Thus, the electron beam evaporated $\mathrm{Cu}_{2} \mathrm{SnSe}_{3}$ thin films showed high purity of structure and good morphological, optical and electrical properties comparable with other methods of thin film deposition.
\end{abstract}

Keywords: $\mathrm{Cu}_{2} \mathrm{SnSe}_{3}$ thin films; electron beam evaporation method; purity in phase structure

(C) Wroclaw University of Technology.

\section{Introduction}

I-IV-VI ternary chalcogenides were traditionally regarded as low or middle band gap semiconductors. These ternary chalcogenide functional materials are suitable for solid lubricant due to their outstanding thermal and mechanical properties. In $\mathrm{I}_{2}-\mathrm{IV}_{-} \mathrm{VI}_{3}$ family, $\mathrm{Cu}_{2} \mathrm{SnSe}_{3}$ (CTSe) have a low melting point and mass density along with high-mean atomic weight and refractive index. Hence, they are identified as a good candidate for acousto-optic applications in the IR region $[1,2]$. CTSe is also a promising thermoelectric semiconductor with relatively high electrical conductivity and moderate thermal conductivity and it may be widely used in industry for small-scale cooling components and low temperature power generators [3]. CTSe compound semiconducting materials with direct optical band gap between 1.1 and $1.5 \mathrm{eV}$ are explored as candidates for absorber layers in thin film heterojunction solar cells [4]. Investigations on thin

*E-mail: kmohanraj.msu@gmail.com films of these compounds are quite relevant for application in thin film waveguides, LEDs, etc. [5]. Recent investigations show that stoichiometric $\mathrm{Cu}_{2} \mathrm{SnSe}_{3}$ crystallizes in a monoclinic structure at low temperature, and in a cubic zinc blende-like (sphalerite) structure at elevated temperatures $[1,6]$.

$\mathrm{Cu}_{2} \mathrm{SnSe}_{3}$ thin films were successfully deposited earlier by using several physical thin film deposition techniques such as flash evaporation [1], laser evaporation, MOCVD [5], co-evaporation [6], thermal evaporation [7], sputtering [8], etc. Among the different physical methods, the use of electron beam deposition method has been investigated for the growth of ternary and quaternary semiconductors by many research groups. This method is advantageous for the control of stoichiometry in alloy films though it also has disadvantages such as equipment cost and use of high voltages. To the best of our knowledge no researchers have deposited $\mathrm{Cu}_{2} \mathrm{SnSe}_{3}$ thin films by this method. Hence, in this work, the authors aimed towards the preparation of $\mathrm{Cu}_{2} \mathrm{SnSe}_{3}$ thin films by electron beam 
evaporation method and the structural, morphological, compositional, optical and electrical characteristics of the films were analyzed.

\section{Experimental procedure}

The electron beam evaporation method is a vacuum deposition process in which target material is evaporated by electron bombardment. First, commercially available $\mathrm{Cu}(0.052 \mathrm{~g}), \mathrm{Sn}(0.050 \mathrm{~g})$ and $\mathrm{Se}(0.098 \mathrm{~g})$ metal powders with high purity $(99.7 \%, 99.5 \%$ and $99.9 \%)$ were taken as target evaporation sources which were placed in a quartz crucible wrapped with a tungsten wire and the ultrasonically cleaned micro-glass substrates were situated above the source. The tungsten filament inside the electron gun was heated to emit electrons forming a beam which was directed toward the target materials. When the electron beam struck the target surface, kinetic energy was transformed by the impact into thermal energy. This energy evaporated the target material. The HIND HIVAC Vacuum Coating Unit (model 12A4-DU) setup along with electron beam gun EBG-PS-3K with an operating voltage of 5 to $7 \mathrm{kV}$ and emission current $\sim 25 \mathrm{~mA}$ were used for the coating process under the chamber pressure of $1 \times 10^{-4} \mathrm{~Pa} . \mathrm{Cu}_{2} \mathrm{SnSe}_{3}$ (CTSe) thin films were simultaneously grown on a series of glass substrates by electron beam evaporation.

The structural characteristics of the deposited films were studied by X-ray diffraction patterns recorded using PAN analytical X'pert PRO diffractometer with $\mathrm{CuK} \alpha$ radiation $(\lambda=0.154 \mathrm{~nm})$. The Raman spectrum was performed at room temperature with LABRAM-HR argon Laser Micro Raman spectrometer of a wavelength of $514 \mathrm{~nm}$. The surface morphology was examined using a field emission scanning electron microscope (FESEM), model BRUKER-QUANTAX EDS equipped with an energy dispersive spectrometer (EDS) at an accelerating voltage of $10 \mathrm{kV}$ to determine the elemental composition of the thin film. The optical measurement was performed by using Hitachi U3400 UV-Vis spectrometer. The electrical study was carried out using Autolab potentiostatgalvanostat model FRNA-32 by applying $\pm 1 \mathrm{~mA}$ current with linear sweep voltammetry galvanostatic mode.

\section{Results and discussion}

\subsection{Structural analysis}

\subsubsection{XRD analysis}

Fig. 1 shows the structure (a) and XRD pattern (b) of an as-deposited CTSe film. The diffraction pattern shows three crystalline peaks at $2 \theta=$ $27.07^{\circ}, 31.09^{\circ}$ and $45.27^{\circ}$ belonging respectively to $\left(\begin{array}{lll}1 & 1 & 1\end{array}\right),\left(\begin{array}{lll}2 & 0 & 0\end{array}\right)$ and $\left(\begin{array}{lll}2 & 2 & 0\end{array}\right)$ planes of a cubic sphalerite-like structure with a space group F-43m. The results are matched well with the standard JCPDS card No 65-7524 which indicates a pure $\mathrm{Cu}_{2} \mathrm{SnSe}_{3}$ compound, whereas the XRD patterns of the films obtained using other physical methods like thermal evaporation, co-evaporation and flash evaporation show $\mathrm{CuSe}_{2}$ and $\mathrm{SnSe}_{2}$ as secondary impurity peaks $[1,5,7]$. The cubic sphalerite-like structure (Fig. 1a) reveals the possible coordination among $\mathrm{Cu}, \mathrm{Sn}$ and $\mathrm{Se}$ atoms. Each anion is coordinated with four cations (Se) located at the corners of a slightly distorted tetrahedron [1].

The average crystallite size of the CTSe film was calculated from Debye-Scherrer formula, $\mathrm{D}=0.9 \lambda / \beta \cos \theta$, where $\lambda$ is the wavelength of $\mathrm{X}$-ray radiation, $\theta$ is the Bragg angle of the peak and $\beta$ is the angular width of the full-width at half maximum (FWHM). Average crystallite size of the CTSe film was found to be $12 \mathrm{~nm}$. This result is in close accordance with the earlier reports on nanoink coated CTSe films $(15.5 \mathrm{~nm})$ [9], whereas it is less than for thermally evaporated thin films with $\mathrm{D}=24.12 \mathrm{~nm}$ [7]. The microstrain $(\varepsilon)$ and dislocation density $(\delta)$ of the CTSe film was calculated according to our earlier report [10] and it were found to be $3.2 \times 10^{-3}$ and $6.94 \times 10^{15} \mathrm{~cm}^{-2}$, respectively. The lattice constant and cell volume of the sample were calculated using UNITCELL software. These were found to be $\mathrm{a}=5.68 \AA$ and $\mathrm{V}=184.20 \AA^{3}$ and were much closer to the standard values of $\mathrm{a}=5.69 \AA$ and $\mathrm{V}=184.80 \AA$ of JCPDS card no 65-7524. 


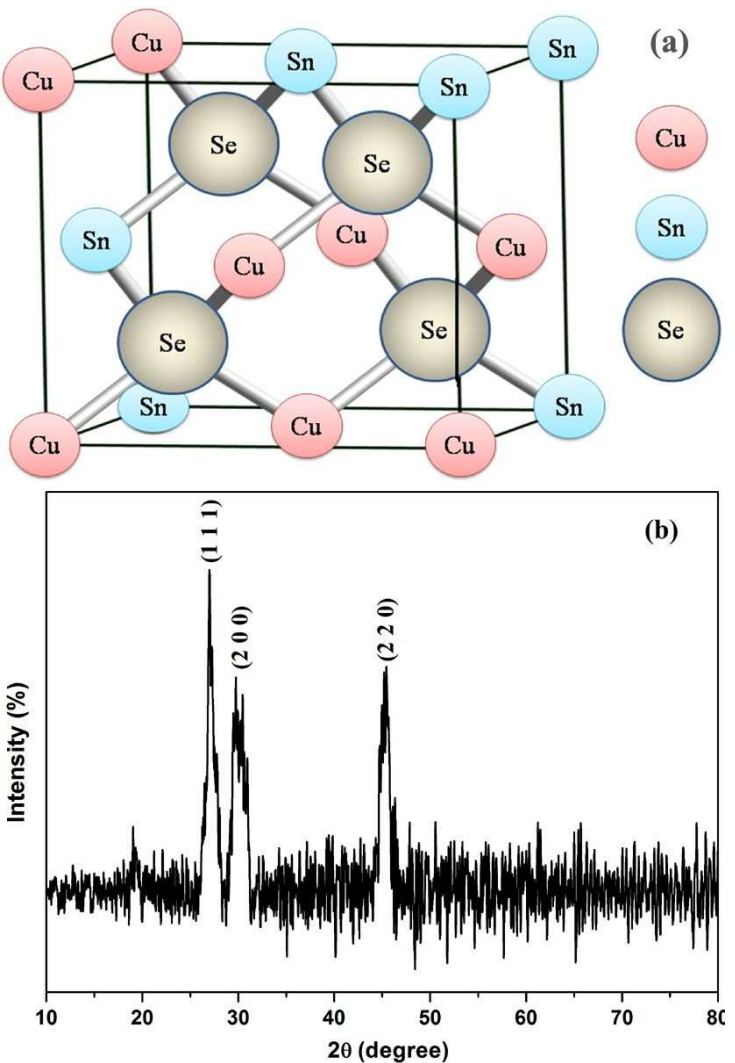

Fig. 1. (a) cubic sphalerite-like structure and (b) XRD pattern of as-deposited $\mathrm{Cu}_{2} \mathrm{SnSe}_{3}$ thin film

\subsubsection{Raman analysis}

The Raman spectroscopy is used as a complimentary tool to know the presence of different phases present in a film. Fig. 2 shows the Raman spectrum of the CTSe thin film at room temperature.

It indicates the appearance of humps corresponding to $\mathrm{Cu}_{2} \mathrm{SnSe}_{3}$ phase at $172,179,189,195$ and $203 \mathrm{~cm}^{-1}$. The result is in agreement with the previous reports on co-evaporated $[4,6]$ and nanoink coated [9] CTSe thin films. The result obviously supports the XRD analysis in which pure $\mathrm{Cu}_{2} \mathrm{SnSe}_{3}$ phase formation was confirmed.

\subsection{Microstructural and compositional analysis}

\subsubsection{FESEM analysis}

The FESEM image (Fig. 3a) shows a continuous film with poly-dispersed grains of diameter less

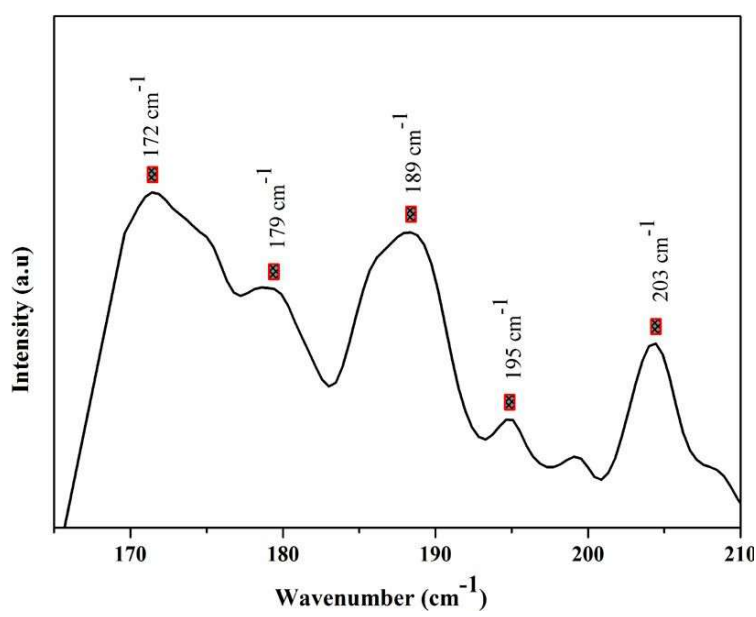

Fig. 2. Raman spectrum of $\mathrm{Cu}_{2} \mathrm{SnSe}_{3}$ thin film

than $1 \mu \mathrm{m}$. The grains are strongly adherent to the substrate. Distinct and very finely dispersed grains evidence for the formation of nucleation centers followed by grain growth and film formation process. Similar morphology was reported for CTSe thin films produced by nanoink coating onto a glass substrate [9] as well as co-evaporated thin films $(0.6 \mu \mathrm{m})$ reported by Suresh Babu et al. [2] and Udhay Bhasker et al. [4].

Fig. $3 \mathrm{~b}$ indicates the compositional analysis of $\mathrm{Cu}_{2} \mathrm{SnSe}_{3}$ thin films. The result indicates that the atomic ratio of $\mathrm{Cu}: \mathrm{Sn}: \mathrm{Se}$ was approximately 1.92:1:2.64. Considering the error of the EDS detector $( \pm 2$ at.\%), the ratio was almost stoichiometric [11]. Thus, the result supports the XRD and Raman results.

\subsection{Optical analysis}

\subsubsection{UV-Vis analysis}

The absorption spectrum of CTSe thin film is shown in Fig. 4a. Relatively high values of absorption coefficient $\left(>10^{4} \mathrm{~cm}^{-1}\right)$ in the visible region (400 to $700 \mathrm{~nm}$ ) indicate that the $\mathrm{Cu}_{2} \mathrm{SnSe}_{3}$ thin film has visible light energy conversion efficiency. The optical band gap of the film with direct allowed transition is fitted by Tauc equation, $\alpha \mathrm{h} v=\mathrm{A}\left(\mathrm{h} v-\mathrm{E}_{\mathrm{g}}\right)^{\mathrm{n}}$ where, $\mathrm{E}_{\mathrm{g}}$ is the band gap, $\mathrm{A}$ is the constant, $\mathrm{h}$ is the Planck's constant and $n$ takes the value of 2 for direct allowed transition. From Fig. 4b, the band gap value was found 


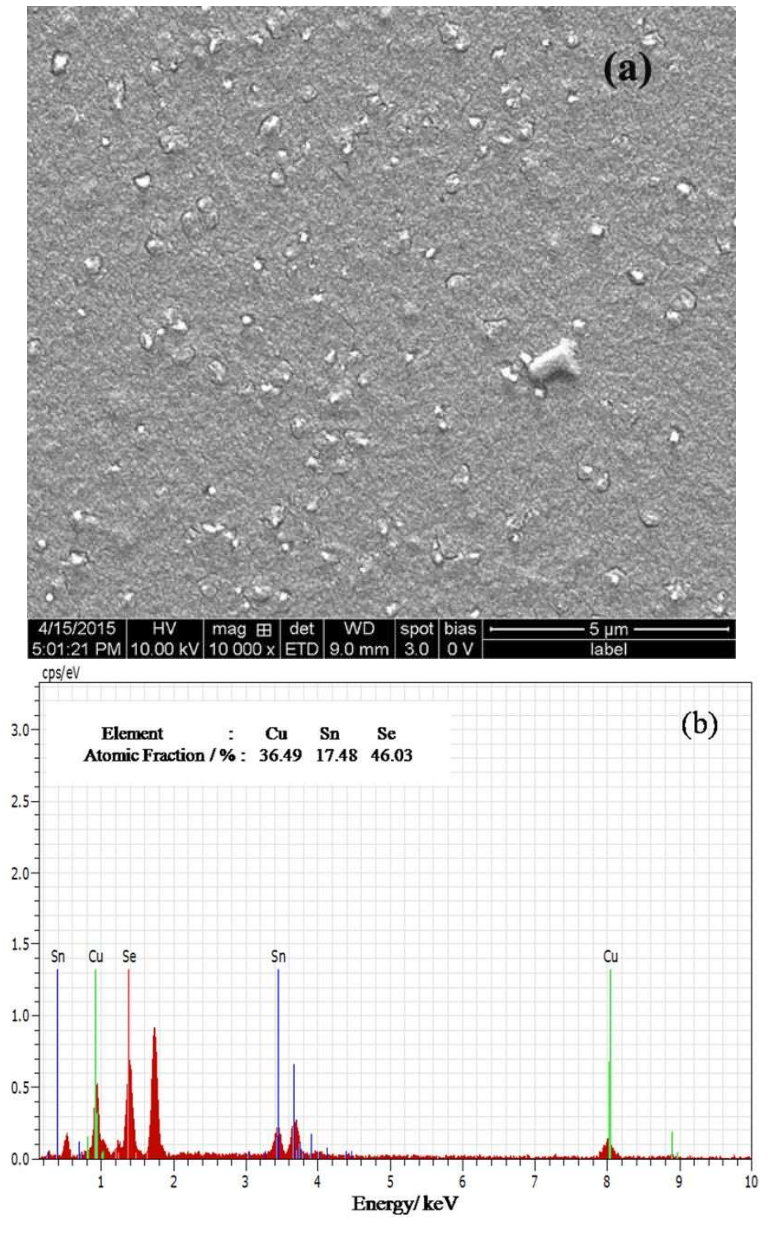

Fig. 3. (a) surface morphology and (b) elemental composition of $\mathrm{Cu}_{2} \mathrm{SnSe}_{3}$ thin film

to be $1.15 \mathrm{eV}$. Hence, the absorption process has been attributed to the optical transition from the acceptor level to the bottom of conduction band due to a free-to-bound transition $[2,7,12]$.

This result is in consistence with the coevaporated CTSe thin films whose direct optical band gap is $1.12 \mathrm{eV}$ [2] but it is less in the case of flash evaporated CTSe thin films with the band gap of $0.84 \mathrm{eV}$ [1], thermal evaporated CTSe thin films with the band gap of $0.94 \mathrm{eV}$ and DC sputtered film whose band gap is about $0.76 \mathrm{eV}$ [8].

\subsection{Electrical study}

The resistivity ( $\rho=$ RA/L) of the CTSe film was calculated by measuring the resistance and physical dimensions of the films and the electrical
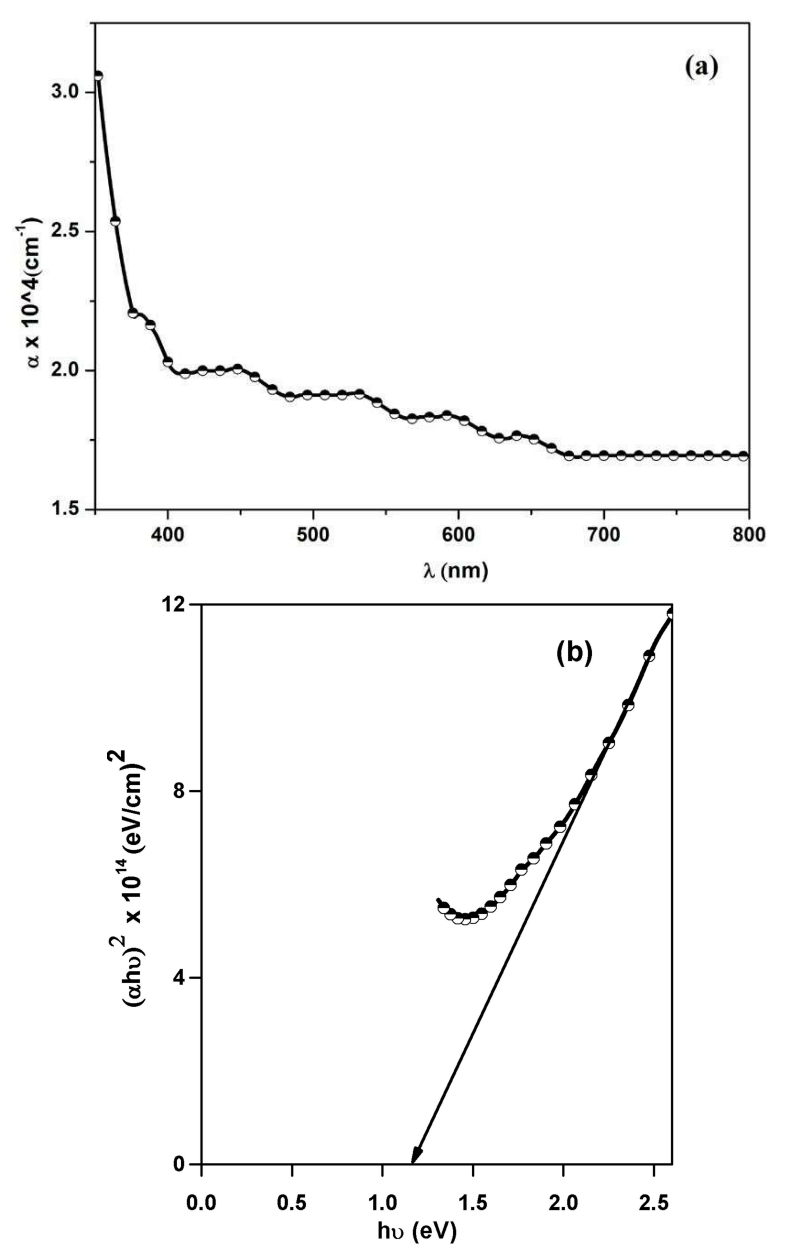

Fig. 4. (a) optical absorption and (b) band gap plot of $\mathrm{Cu}_{2} \mathrm{SnSe}_{3}$ thin film

conductivity $(\sigma)$ was calculated based on the expressions and $\sigma=1 / \rho$. The values of resistivity and conductivity of the film were determined using the slope value of the I-V (Fig. 5) plot and were found to be $2.13 \Omega \cdot \mathrm{cm}$ and $0.468 \mathrm{~S} / \mathrm{cm}$ respectively.

The electrical resistivity is slightly higher when compared with thermally evaporated thin films $(1.01 \Omega \cdot \mathrm{cm})$ [7], sputtered thin films $(0.12 \Omega \cdot \mathrm{cm})[11]$ and the films obtained by nanoink coating $(0.25 \Omega \cdot \mathrm{cm})$ [9]. But the resistivity is less than that of the flash evaporated thin film (16 to $124 \Omega \cdot \mathrm{cm}$ ) [1]. Formation of larger nucleation centres in the evaporated film results in small crystallite sizes which ultimately increase the intercrystalline barriers and this may be responsible for the increase in electrical resistivity [7]. 


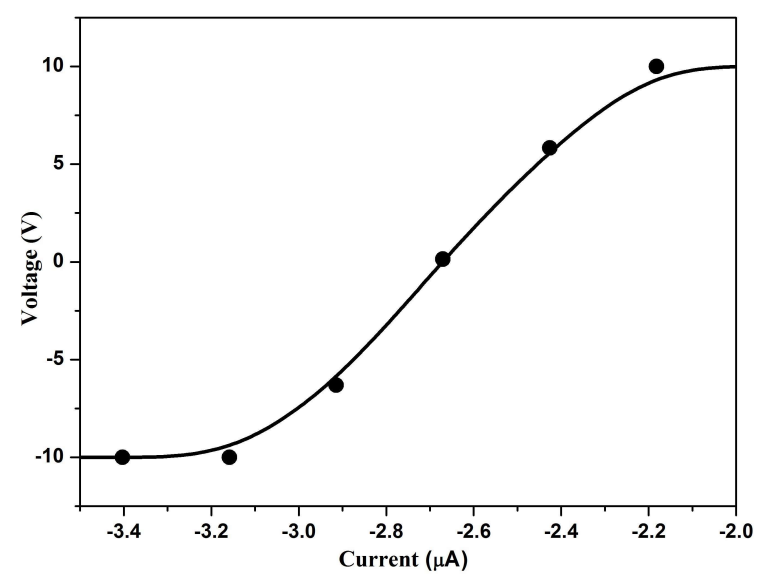

Fig. 5. I-V characteristics plot of $\mathrm{Cu}_{2} \mathrm{SnSe}_{3}$ thin film

\section{Conclusions}

In summary, CTSe thin films deposited by electron beam evaporation method show pure phase structure when compared with CTSe thin film obtained by other methods of deposition. The morphological, optical and electrical properties of the films obtained by this method are highly comparable with those obtained by other physical methods. Hence, the good structural and highly desirable optical and electrical properties of the electron beam evaporated $\mathrm{Cu}_{2} \mathrm{SnSe}_{3}$ thin films make them a prospective candidate for solar cell applications.

\section{Acknowledgements}

The authors gratefully acknowledge FIST, UGCSAP, New Delhi for providing the financial support to the Department of Physics, Manonmaniam Sundaranar University, Tirunelveli, Tamil Nadu, India and the Professor and Head of Department of Nano Science and Technology, Bharathiyar University for the help in FESEM-EDS analysis and Sri Ramakrishna Engineering College for providing the facilities of UV-Visible and I-V characterizations.

\section{References}

[1] Hema Chandra G., Lakshmana Kumar O., Prasada Rao R., Uthanna S., J. Mater. Sci., 46 (2011), 6952.

[2] Suresh Babu G., Kishore Kumar Y.B., Bharath Kumar Reddy Y., Sundara Raja V., Mater. Chem. Phys., 96 (2006), 442.

[3] Song J.M. et al., J. Alloys Compd., 581 (2013), 646.

[4] Uday Bhaskar P., Suresh Babu G., Kishore KUMAR Y. B., Sundara Raja V., Appl. Surf. Sci., 257 (2011), 8529.

[5] Ibanez M., Cadavid D. et al., J. Mater. Chem. A, 1 (2013), 1421.

[6] Kim K.M., Tampo H., Shibata H., Niki S., Thin Solid Films, 536 (2013), 111.

[7] Hamdani K. et al., Opt. Mater., 37 (2014), 338.

[8] Kuo D.-K., Haung W.-D., HuAnG Y.-S., Wu J.-D., LIN Y.-J., Thin Solid Films, 518 (2010), 7218.

[9] LeE P.-Y., Shei S.-C, Hsu E.-H., Chang S.-J., Mater. Lett., 102 (2013), 120.

[10] Henry J., Mohanraj K., Kannan S., Barathan S., Sivakumar G., J. Optoelect. Adv. Mater, 15 (2013), 1047.

[11] ZhU L., QIANG Y.-H., Zhao Y.-L., Gu X.-Q., Song D.-M., Song C.-B., Acta phys. Chem. Sin., 11 (2013), 2339.

[12] Zulkarnain Z., Anuar K., Mohd Z.H., Chuah H.C., Mater. Lett., 58 (2004), 2199.

Received 2015-10-09

Accepted 2016-09-09 